NASA Technical Memorandum 103679

AIAA-91-0273

\title{
Improved Visualization of Flow Field Measurements
}

Jeffrey Hilton Miles

Lewis Research Center

Cleveland, Ohio

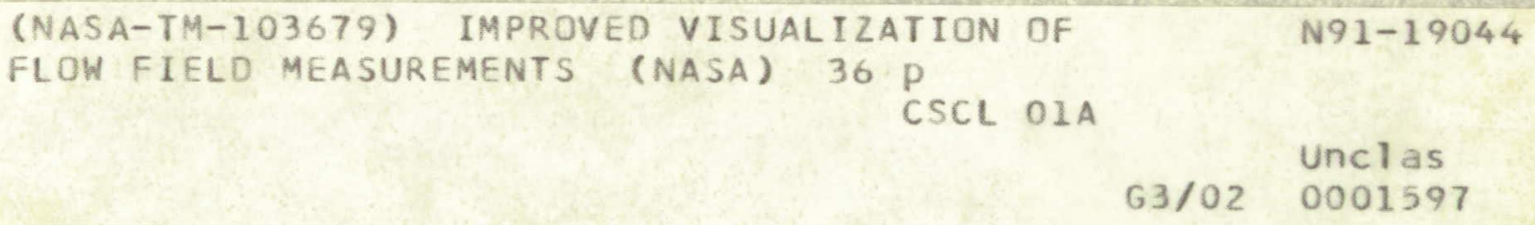

Prepared for the

29th Aerospace Sciences Meeting sponsored by the American Institute of Aeronautics and Astronautics Reno, Nevada, January 7-10, 1991 


\author{
Jeffrey Hilton Miles \\ National Aeronautics and space Administration \\ Lewis Research Center \\ Cleveland, Ohio 44135
}

Summary

A capability has been developed that makes it possible to apply to measured flow field data the visualization tools developed to display numerical solutions for computational fluid dynamic problems. The measurement monitor surface (MMS) procedure was applied to the analysis of flow field measurements within a low-aspect-ratio transonic axial-flow fan rotor obtained with 2-dimensional laser anemometry. The procedure generates input for the visualization tools developed to display numerical solutions for computational fluid dynamic problems. The relative Mach number contour plots obtained by this method resemble the conventional contour plots obtained by more traditional methods. The results demonstrate that the MMS procedure can be used to generate input for the multidimensional processing and analysis tools developed for data from numerical flow field simulations. They show that an experimenter can apply the MMS procedure to his data and then use an interactive graphics program to display scalar quantities like the Mach number by profiles, carpet plots, contour lines, and surfaces using various colors. In addition, flow directionality can be illustrated by display of vector fields and particle traces. The MMS procedure thus has the potential to produce better insight into turbomachinery and other types of fluid flow physics.

\title{
Introduction
}

Flow visualization is an important experimental technique used to study complex physical phenomena. Data sets obtained are images, well suited 
to 2-dimensional image processing and analysis. Flow visualization is also an important tool used to deal with the vast amount of data generated by numerical simulations. Numerical simulations produce multidimensional data that require additional processing and analysis tools to display the information in a form that leads to a better understanding and evaluation of simulated flows. A computational fluid dynamic researcher at a graphics workstation can display various portions of a computed flow field, observe the solution from different vantage points, and interact with the computed solution. ${ }^{1-3}$ Having the ability to interact with the solution provides an opportunity to discover information that might not be anticipated.

The vast amount of data generated by numerical simulations matches the flow of data from experiments using advanced instrumentation such as laser anemometry. It would be advantageous if the multidimensional processing and analysis tools developed for data from numerical simulations were available to be used for multidimensional experimental data. Then an experimenter using an interactive graphics program could display scalar quantities like the Mach number by profiles, carpet plots, contour lines, and surfaces using various color intensities. In addition flow directionality could be illustrated by displaying vector fields and particle traces. This capability would provide the experimenter with the same opportunity as a computational fluid dynamic flow researcher to discover information that might not be anticipated.

This paper focuses on the application of a flow visualization scheme developed to aid the analysis of numerical data from fluid flow simulations to experimental data from laser anemometer measurements made in a transonic axial-flow fan rotor ${ }^{4,5}$ The measurements consisted of two components of velocity measured at many points in the fan flow velocity field. These measurements were made as part of an extensive experimental, theoretical, and computational turbomachinery research program being. conducted at the NASA Lewis Research Center. 
Several problems were encountered in the process of applying the flow visualization schemes used on results from fluid dynamic simulations to experimental data. One problem was the poor resolution of the fan blade location when the flow visualization was applied to the experimental data. A second problem was due to the relatively low density of the measurement points in the flow field. The problems were dealt with simultaneously by properly enriching the measured data set through the application of a measurement monitor surface (MMS) procedure. In the following sections, the MMS procedure will be described, and results of the procedure will be shown and discussed.

\section{Nomenclature}

AP

LA axial position, 2

$a_{\ell}$

local speed of sound

a speed of sound based on plenum total temperature

CP

LA circumferential position, $\theta$

$c_{p}$

specific heat at constant pressure

$d_{k}$

distance between interpolation point and data point, Eq. (6)

$F(x, y)$ interpolated value at $x, y$

$f_{k} \quad$ measured value at point $x_{k}, Y_{k}$

$J$

Jacobian of transformation, Eq. (2)

$M_{\text {rol }}$ relative Mach number, Eq. (16)

$M_{w}$ molecular weight of air, $0.02897 \mathrm{gm} /(\mathrm{kgm}$ mole)

n polytropic exponent

P,Q grid cluster control functions, Eq. (1) 


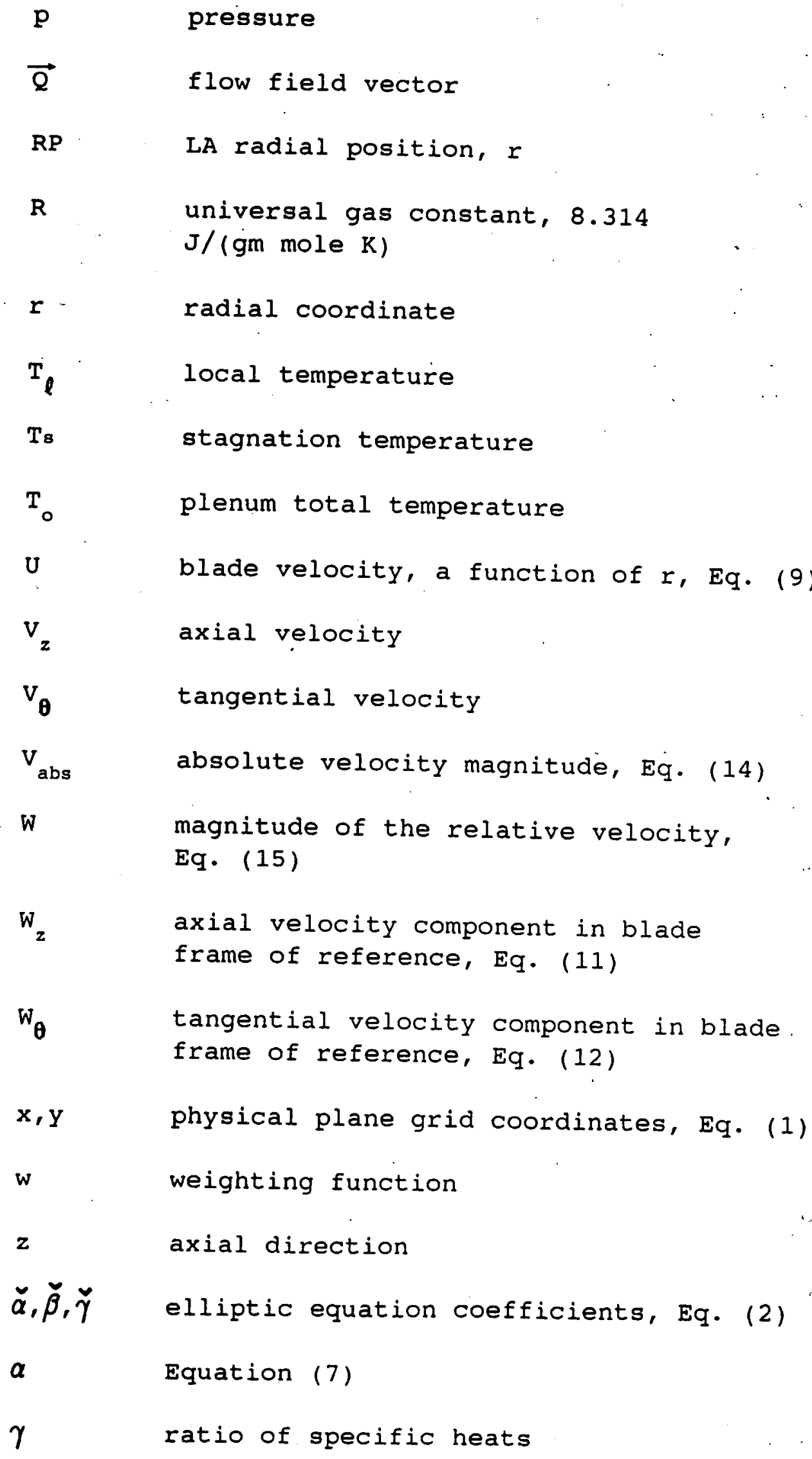




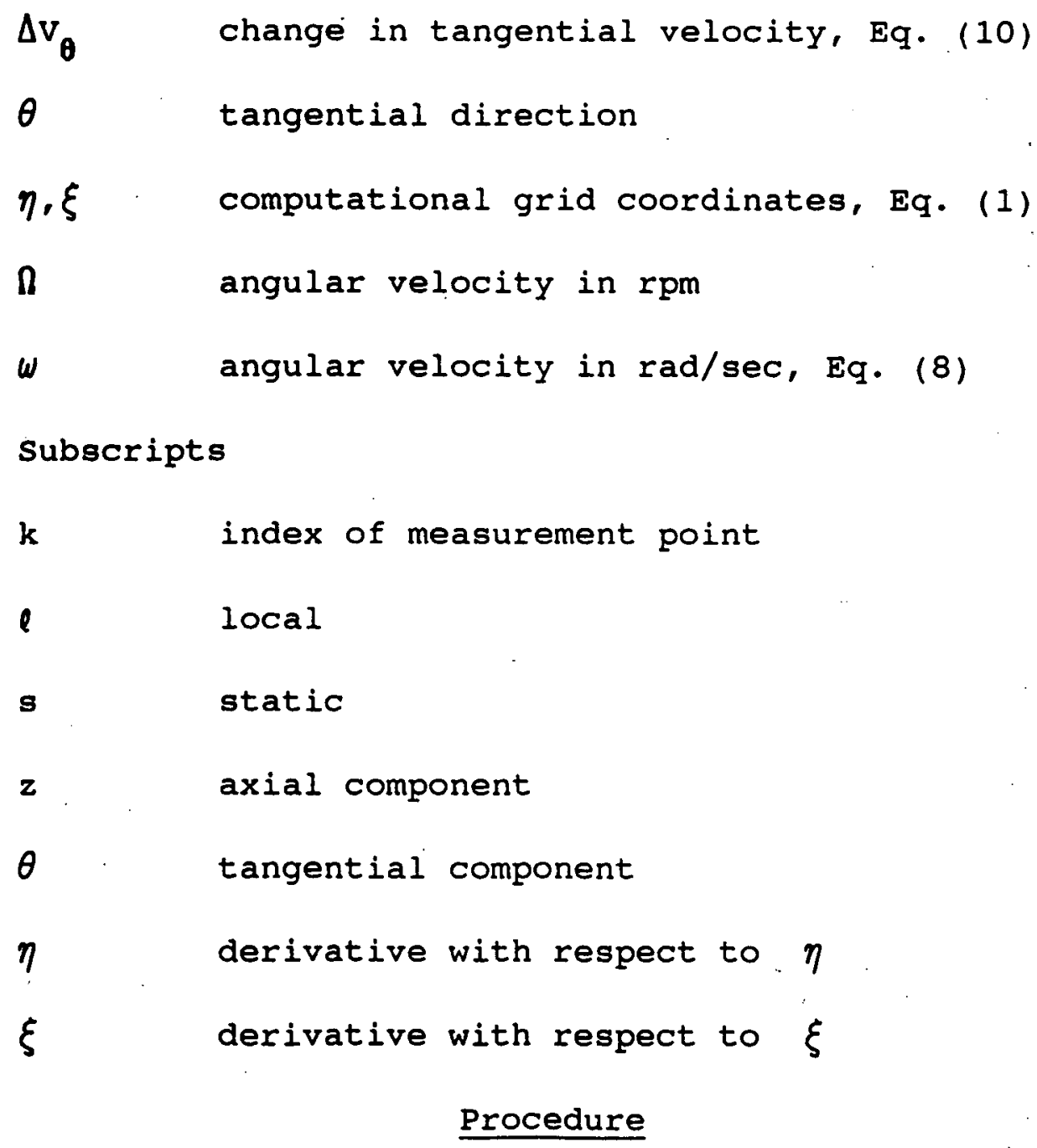

The procedure discussed herein was developed for a specific experimental data set. The experimental data set chosen is reported in Refs. 4 and 5. It consists of two components of velocity measured at many points in the flow field of a transonic axial-flow fan rotor. The measurements were made using a laser anemometer. While the procedure discussed herein can be used to generate input for any numerical flow field visualization methodology, the one used for development is PLOT3D which is described in Refs. 1 to 3.

As a first step in developing the capability to apply to measured flow field data the visualization tools developed to display numerical flow field solutions, a simple flow physics model was used to generate density, two components of momentum, and energy at each point in the measurement 
grid. The flow physics model was based on the premise that the flow field data were acquired using laser anemometer measurements along surfaces that approximate the location of the rotor design streamlines. The flow physics model is described in Appendix A. A description of the procedure for calculating the density, the two-dimensional momentum, and energy is given in Appendix B.

In looking at the display of the results using data at the measurement points only, the first problem observed was that the measurements did not clearly define the axial-flow fan rotor geometry. The presence of a rotor was indicated by the absence of data. Data were taken at only 30 positions in the axial projection. Only 10 positions were inside the rotor. Fifty positions were used in the circumferential projection. Hence, the rotor was defined by the absence of data at only about 10 points in the axial. direction, and thus the rotor appeared jagged and nonaerodynamic.

The second problem was that the density of measurement points in the axial direction was low so that the contour routine in the flow visualization program produced notched contours. Both of these problems were dealt with simultaneously by the measurement monitor surface (MMS) procedure which will be described next.

In spite of these discouraging results, the simple flow physics model is the basis of the MMS procedure, and the first step in the MMS procedure is to use a simple flow physics model to generate. the density, the 2-dimensional momentum, and energy. at each measurement point in a coordinate frame fixed with the axial-flow fan rotor (Appendices $A$ and $B$ ).

The procedure uses the following information from the experiment:

1. Measurements of axial-flow fan rotor geometry 
2. Laser anemometry data that consists of the axial velocity, $\mathrm{V}_{\mathbf{z}^{\prime}}$, and tangential velocity, $v_{\theta^{\prime}}$ and the coordinates of all the measured points

3. Test condition data (the axial-flow fan rotor velocity, inlet static pressure, inlet stagnation temperature)

The second step in the procedure is to use a simple grid generation scheme to derive a monitor surface grid that includes the blade shape, employs streamline-type coordinates, and encompasses all the points in the measurement grid. The axial-flow fan rotor geometry and the measurement grid scheme information are used to generate the new grid. The grid generation scheme used will be discussed in the following section. The third step in the procedure is the use of a scattered data interpolation scheme known as shepard's method to interpolate the density, the two components of momentum, and the energy values from the measurement grid to the new monitor surface grid. The grid interpolation procedure is discussed in more detail in the section on grid interpolation. The fourth step in the procedure is to use the visualization tools to display the monitor surface density, momentum and energy fields, and quantities that can be calculated from them. In practice, the results are displayed using PLOT3D on an Silicon Graphics IRIS workstation. 6

\section{Grid generation}

The requirements of the monitor surface grid were that it include the blade shape, employ streamline-type coordinates, and encompass all the points in the measurement grid. Many grid generation techniques might meet these requirements. The grid generation procedure used in this investigation is discussed in Refs. 7 to 10 .

The computer code used to generate the new grid employed the same logic as the code presented in Ref. 8. However, the FORTRAN code was adapted to run on a VAX-CLUSTER and modifications were made 
in places like the data input and data output schemes and the size of the arrays.

The grid generation scheme involves a mapping from a computational plane $(\xi, \eta)$ to a physical plane $(x, y)$. The mapping is constructed by specifying the desired grid points on the boundary of the physical domain. The grid is generated by solving a Poisson partial differential equation in the form

$$
\begin{aligned}
& \frac{\partial^{2} \xi}{\partial x^{2}}+\frac{\partial^{2} \xi}{\partial y^{2}}=P(\xi, \eta) \\
& \frac{\partial^{2} \eta}{\partial x^{2}}+\frac{\partial^{2} \eta}{\partial y^{2}}=Q(\xi, \eta)
\end{aligned}
$$

(where $P$ and $Q$ are known functions used to control interior grid clustering.

These equations are transformed to computational space by interchanging the roles of the variable $(\xi, \eta)$ and $(x, y)$.

This yields the following set of elliptic equations:

$$
\begin{aligned}
& \check{\alpha} x_{\xi \xi}-2 \check{\beta} x_{\xi \eta}+\check{\gamma} x_{\eta \eta}=-J^{2}\left(P x_{\xi}+Q x_{\eta}\right) \\
& \check{\alpha} y_{\xi \xi}-2 \check{\beta} y_{\xi \eta}+\check{\gamma} y_{\eta \eta}=-J^{2}\left(P y_{\xi}+Q y_{\eta}\right)
\end{aligned}
$$

where

$$
\begin{aligned}
& \check{\alpha}=x_{\eta}^{2}+y_{\eta}^{2} \\
& \check{\beta}=x_{\xi} x_{\eta}+y_{\xi} y_{\eta} \\
& \check{\gamma}=x_{\xi}^{2}+y_{\xi}^{2} \\
& J=\frac{\partial(x, y)}{\partial(\xi, \eta)}=x_{\xi} y_{\eta}-x_{\eta} y_{\xi}
\end{aligned}
$$

Numerical computations are performed in the uniform rectangular transformed plane, $(\xi, \eta)$, to solve Eq. (2). The solution provides the $(x, y)$ coordinates of each point in physical space. The axial-flow fan rotor geometry was used in a simply connected region mapping. For a simply connected region, Dirichlet boundary conditions can be used at all boundary points. 
The measurement grid is 3-dimensional and is represented in radial, $r$ (or RP), circumferential, $\theta$ (or $\mathrm{CP}$ ), and axial, $\mathrm{z}$ (or AP), coordinates. The $(r, \theta, z)$ coordinates are used in analytical expressions, while the ( $R P, C P$, and $A P$ ) coordinates are used in describing the experimental results discussed in Refs. 4 and 5 . However, the data were principally collected in the cascade plane which is a plane defined by $z$ (or AP) and $\theta$ (or RP) coordinates for nearly constant $r$ (or RP). The measurement grid thus obtained is unrolled by the transformation

$$
\begin{aligned}
& x=z \\
& y=r(z) \theta
\end{aligned}
$$

The transformation (Eq. (4)) applies to the monitor surface grid so that the physical space $(x, y)$ is actually $(z, r(z) \theta)$. In addition, it is used in the grid interpolation procedure that will be discussed next.- Consequently, here also the physical space $(x, y)$ is actually $(z, r(z) \theta)$.

Use of Dirichlet boundary conditions means the solution must have prescribed values on the boundary. Considering a rectangular region in the $(\eta, \xi)$ space, the boundary conditions are specified as follows. The inflow circumferential boundary condition is applied on the left side of the rectangle and the outflow circumferential boundary condition is applied on the right side. The blade suction surface is defined on the top of the rectangle and the blade pressure surface is defined on the bottom of the rectangle. The nonlinear simultaneous algebraic equations (Eq. (2)) were solved iteratively using a successive over-relaxation technique (SOR).

\section{Grid Interpolation}

Interpolating the values of density, momentum, and energy from the measurement grid to the monitor surface grid is not trivial. Many schemes of curve and surface fitting are available. ${ }^{11}$ However, most of these schemes assume the data points lie on a complete rectangular lattice in the 
xy-coordinate plane and function values (and possibly slopes) are available at each point. In the case under consideration, the points are not assumed to satisfy any particular conditions as to spacing and density. Consequently, they are referred to as being "scattered." scattered data interpolation schemes are reviewed in Refs. 12 to 14 .

Because of its simplicity, the scattered data interpolation technique used was shepard's method. ${ }^{12-14}$ This method is based on the premise that the value of a function $F(x, y)$ at a point $(x, y)$ in the monitor surface grid will be similar to the value of a point $f_{k}$ in the measurement grid if the points $(x, y)$ and $\left(x_{k}, y_{k}\right)$ are close. Since the point in the monitor surface grid can be near many points in the measurement grid, the value of $F(x, y)$ determined using shepard's method is given by a weighted average as follows:

$$
F(x, y)=\frac{\sum_{k=1}^{N} w_{k}(x, y) f_{k}}{\sum_{k=1}^{N} w_{k}(w, y)}
$$

where the weights are a function of the distance between the points and decrease as the distance between points increases. As a result, measurement points at large distances from a monitor surface point do not influence its value significantly.

In this Equation, $F(x, y)$ represents the density, momentum, or energy in the new coordinate system, $f_{k}$ represents the density, momentum, or energy in the old coordinate system (measurement grid) $\left(x_{k}, y_{k}\right)$, and $N$ is the number of points used in the weighted average.

The scattered data interpolation works as follows. Each point in the new grid is first located relative to a cell defined by the old grid. While it is preferable that the point in the new grid be in a cell defined by the old grid, it is 
not necessary. This method has the distinct advantage that the point in the new gird need not be in a cell defined by the old grid. The four corners of this cell are used in the interpolation ( $N=4)$. The procedure provided for a choice of weight, $w_{k}(x, y)$, as either $d_{k}^{-1}, d_{k}^{-2}$, or $e^{-\alpha \sigma_{k}^{2}} d_{k}^{-2}$ where the distance between points is defined by

$$
d_{k}=\sqrt{\left(x-x_{k}\right)^{2}+\left(y-y_{k}\right)^{2}}
$$

and

$$
\alpha=\frac{N}{\sum_{k=1}^{N} d_{k}^{2}}
$$

This value of $\alpha$ is selected to reduce the influence of far away points.

The first derivatives of $F(x, y)$ vanish at the data points $\left(x_{k}, y_{k}\right)$. As a result, $F(x, y)$ is flat near the data points.

Shepard's method of scattered data interpolation was tried with each of the weights discussed in this section. No visual change was apparent in the results.

\section{Implementation}

To implement the measurement monitor surface (MMS) procedure one must write computer code. Consequently, one must have some experience with several disjoint engineering disciplines so that everything can be blended together. The MMS that makes it possible to apply to measured axial-flow fan rotor flow field data the visualization tools developed to display numerical solutions from computational fluid dynamic problems is based on a blend of knowledge from four fields. The first component of the procedure is a simple flow physics model based on the premise that the flow field data were acquired using laser anemometer measurements along surfaces that approximate the location of the rotor design streamlines. Using this physical 
model, information about the test condition, and the measured data, the procedure generates a density, 2-dimensional momentum, and energy at each measurement point in a coordinate frame fixed with the axial-flow fan rotor. The second component of the procedure is a grid generation scheme that is used to derive the monitor surface grid that includes the blade shape, has streamline-type coordinates, and includes the points in the measurement grid (grid generation section). The third component of the procedure is the use of a scattered data interpolation scheme known as Shepard's method to interpolate the density, the two components of momentum, and the energy values from the measurement grid to the new monitor surface grid (grid interpolation section). The fourth component of the procedure is the visualization tools that are used to visualize the monitor surface and quantities that can be calculated from them.

In practice, the results were displayed using PLOT3D on an Silicon Graphics IRIS workstation. ${ }^{6}$ Consequently, some familiarity with the PLOT3D manual is also needed. In addition, one needs to understand certain aspects of the turbomachinery experiment such as the laser anemometry measurement scheme and the axial-flow fan geometry.

The flow field calculations and the use of PLOT3D are discussed in Appendices $A$ and $B$. The flow logic of the computer programs developed to implement the procedure is discussed in Appendix $C$.

\section{$\underline{\text { Results }}$}

In this section, the measurement monitor surface procedure will be demonstrated. In addition, a relative Mach number (Appendix A) plot obtained using the MMS procedure and the PLOT3D program will be compared to a plot of the same data made using conventional methods. The experiment. will be discussed first. Then results obtained with the MMS procedure and the PLOT3D flow visualization program will be presented. 


\section{The Experiment}

Although the PLOT3D program makes it possible to view many variables, in this paper the emphasis is on the shock structure. This is displayed in plots of the relative Mach number (Appendix A). The shock structure to be shown is from measurements acquired in a low-aspect-ratio transonic axial-flow fan rotor. Much of the total pressure rise which occurs in advanced fans and core compressor inlet stages occurs across the rotor passage shock. Consequently, these measurements were made to help develop accurate models of the passage shock that could be used in the axial-flow fan rotor design process. Details of the experiment and the results of the test program are discussed in Refs. 4 and 5. The rotor aspect ratio is 1.56 and the design tip relative Mach number is 1.38 . The flow field shown is for a near-stall operating. condition. The plots are based on intrablade velocity measurements acquired with a laser fringe anemometer on blade-to-blade planes in the supersonic region at 30 -percent span.

A schematic diagram of the NASA Lewis singlestage axial-flow compressor test facility is shown in Fig. 1. The facility is discussed in Ref. 4. The test rotor, NASA rotor 67 , is shown in Fig. 2 . The rotor is also discussed in Ref. 4.

The laser anemometer (LA) measurement location in the stationary reference frame is specified by the parameters AP, RP, and CP. As shown schematically in Fig. 3 , AP is the axial position, $\mathrm{RP}$ is the radial position measured from the compressor axis of rotation, and CP the circumferential position measured relative to the $z-\theta-p l a n e$. Velocity measurements occurred along the circumferential measurement line which is swept through the LA measurement location as the blades rotate past the measurement position. The 2-dimensional surface of interest is the $(x, y)$ plane which corresponds to the $(z, r(z) \theta)$ plane. Detailed information on the IA measurements is given in Ref. 4. 
Laser anemometer axial measurement locations (AP) and radial measurement locations (RP) are shown in the meridional view of the test fan rotor presented in Fig. 4. The laser anemometry results presented were obtained at the 30-percent span location shown in Fig. 4. Radial surveys of total and static pressure, total temperature, and flow angle were made at survey stations 1 and 2 located upstream and downstream of the rotor as shown in Fig. 4 .

Figure 5 from Ref. 4 shows a contour plot of the relative Mach number at a near-stall flow test condition at 30-percent span from the shroud produced by a nonworkstation graphical procedure. The MMS procedure was applied to the data measured at this same test condition. Since the same relative Mach number calculation procedure is used and the same data were used, it expected that the MMS procedure results will be similar to the ones shown in Fig. 5.

\section{The MMS Procedure}

Figure 6 shows the grid used for the laser anemometer velocity measurements. The grid has 30 horizontal divisions and 100 vertical divisions. Note that the axial-fiow fan rotor shape defined by the absence of data in the measurement grid has a jagged appearance. The grid generated by the MMS procedure for this example is 51 by 102 and it is shown in Fig. 7 .

Figure 8 shows the measured relative Mach number in the measurement grid. Each shade of color represents a region of constant Mach number. Note the jagged appearance of the axial-flow fan rotor which is an artifact of the measurement scheme and a significant distracting influence. In addition, the low density of points (30) in the axial direction may be hindering the contour drawing package. After viewing these results, the MMS procedure was proposed as a possible solution to the problems shown in this figure. 
The MMS procedure results are shown in Fig. 9. Note that the axial-flow fan rotor shape is now smooth and the contours are smoother.. Figures 10 and 11 show closeups of the measured and MMS procedure-derived relative Mach number contours. As expected, the MMS procedure results are similar to those presented in Fig. 5 .

Looking at the plot of the grid produced by the MMS procedure used herein which was made using the PLOT3D flow visualization program, the shape of the ends of the axial-flow fan rotor are not shown as being as sharp as one expects. The problem is related to the boundary conditions specified in the $(\xi, \eta)$ plane. The $(x, y)$ coordinates along the blade pressure surface are specified on the bottom $\xi$ axis as boundary conditions. The $(x, y)$ coordinates along the blade suction surface are specified on the top $\xi$ axis as boundary conditions. The tips of the blade were specified by three points on the pressure surface and three points on the suction surface. The points specifying the ends are close together while those along the blade are spaced much further apart. This spacing problem caused a great amount of grid distortion at the blade ends. In addition, the distortion was enhanced by the PLOT3D visualization program. While the grid used was adequate for demonstrating the MMS procedure, it is obvious that further refinement is necessary. A more detailed investigation of the grid distortion problem at the ends of the blade would lead to resolution of this problem. However, it might require using a second grid program to adjust the grid points at the blade tips.

A different type of distortion occurs in the flow field at the downstream ends of the axial-flow fan rotor. Here the elongated low Mach number region at the end of the rotor in Fig. 8 is now a sequence of oval low Mach regions in Fig. 9. The shape of the elongated low Mach number region is attributed to a lack of data in this region. The sequence of oval low Mach regions is an artifact of the scheme which tries to interpolate the measurements onto a fine grid. 
While only relative Mach results are shown, all the standard 2-dimensional flow quantity calculations that the PLOT3D program can make are available. These results thus indicate that an experimenter can apply the MMS procedure to his data and then use an interactive graphics program to display scalar quantities like the Mach number by profiles, carpet plots, contour lines, and surfaces using various colors. In addition, flow directionality can be illustrated by display of vector fields and particle traces. The MMS procedure thus has the potential to produce better insight into turbomachinery and other types of flow physics.

\section{Discussion}

Graphical techniques are used by experimenters and computational fluid dynamic researchers to provide an understanding of flow field phenomena and the effects of changes in configuration. In addition, computational fluid dynamic researchers use graphic displays to assess the accuracy of a calculation, and to evaluate errors, inconsistencies, or improper modeling.

The results presented herein suggest experimenters could interactively find a new use for graphical methods since they could assess the usefulness of a particular measurement grid in obtaining data for code validation by using the techniques presented herein to move computational flow field predictions into a measurement grid. Viewing computational results can indicate regions of large gradients where more points are desirable. Simulated experimental results for a particular measurement grid scheme would supply additional information on the the shape of the contours for physical quantities such as relative Mach number.

This technique could also be used during an experimental research program to allow experimenters to monitor interactively their results in a display scheme that included the experimental geometry while the data were being collected. 
Computational fluid dynamic researchers often need to use overlapping grids. The results presented herein suggest they could use shepard's method to interpolate between these grids. This might be especially useful for grids adjusted by direct interaction or grids adjusted during computation.

\section{Conclusion}

A capability has been devloped that makes it possible to apply to measured flow field data the visualization tools developed to display numerical solutions from computational fluid dynamic problems. The procedure is based on a blend of material from four fields of knowledge. First, a simple flow physics model is used to generate density, two components of momentum, and energy at each point in the measurement grid. The flow physics model is based on the premise that the flow field data were acquired using laser anemometer measurements along surfaces which approximate the location of the rotor design streamlines. Second, a simple grid generation scheme is used to derive a grid which includes the axial-flow fan rotor shape and streamline-type coordinates for all the points. in the measurement grid. Third, a scattered data interpolation scheme known as shepard's method is used to move information from the measurement grid to the new grid forming a monitor surface for density, the two components of momentum, and energy. Finally, tools developed to visualize numerical fluid flow results are used to visualize the monitor surfaces and quantities that can be calculated from them.

The measurement monitor surface procedure (MMS procedure) has been demonstrated using laser anemometry measurements in a transonic axial-flow fan rotor made at a near-stall flow test condition at a position 30-percent of the span from the shroud. The Mach number results obtained by the MMS procedure for this case are similar to results obtained using standard techniques. 
These results indicate that a MMS procedure can be used to generate input for the multidimensional processing and analysis tools developed for data from numerical flow field simulations. They show that an experimenter can apply an MMS procedure to his data and then use an interactive graphics program to display scalar quantities like the Mach number by profiles, carpet plots, contour lines, and surfaces using various colors. In addition, flow directionality can be illustrated by display of vector fields and particle trances. The MMS procedure thus has the potential to produce better insight into turbomachinery and other types of flow physics.

\section{Appendix A - Flow Calculations}

$\dot{A}$ procedure for putting laser anemometer (LA) flow field measurements in a transonic axial-flow fan rotor into a form suitable for display in the measurement grid by PLOT3D is discussed.

One limitation of the procedure is that it is assumed that the results from the LA measurements have been acquired along measurement surfaces which approximate the location of the rotor design streamlines. The approximation is clearly incorrect when the grid points are inside a blade surface.

In the analysis of rotor flow and energy transfer, the variables of concern.are of interest in a coordinate system rotating with the blade. In this section the relationships used to convert the measured axial and tangential velocity to the rotating coordinate system will be set forth.

Basic thermodynamic equations used herein such as definitions of specific heat, the speed of sound, the perfect gas law, work done by the system, and the energy equaton are given in thermodynamic text books such as Ref. 15 and in discussions of turbine design (Refs. 16 and 17) and these expressions are not repeated. 
The velocity vector relationships used are presented here since the data are shown in a frame of reference rotating with the blade while it is measured in a stationary laboratory frame of reference. However, turbine design textbooks such as Refs. 16 or 17 should be consulted for more information.

\section{Velocity Vectors}

The angular velocity in rad/sec is related to the velocity in $\mathrm{rpm}$ as follows:

$$
|\omega|=2 \pi \Omega / 60
$$

where

$|n|$ is the velocity in $\mathrm{rpm}$.

For this case $|n|=16042 \mathrm{rpm}$ and $|\omega|=1679.9 \mathrm{rad} / \mathrm{sec}$.

For the system under consideration the following conventions are used. The angular velocity is taken to be negative.

The blade velocity is

$$
U(r)=-I(z)|\omega| \text {. }
$$

For this case with $\mathrm{r}=21 \mathrm{~cm}$, the blade velocity is $-352 \mathrm{~m} / \mathrm{s}$.

The change in tangential velocity is

$$
\Delta V_{\theta}=\left(V_{\theta}\right)_{\text {inlet }}-\left(V_{\theta}\right)_{\theta x i t}
$$

The laser measures two components of an absolute velocity. An axial component, $v_{z^{\prime}}$ and a tangential component $v_{\theta}$. For flow in and across the stators, the absolute velocites are of interst.

For flow in and across the rotors, the velocities are considered relative to the velocity of 
the rotating blades. In terms of relative velocities, flow in a rotating blade row cán be analyzed in a manner similar to the analysis of flow in a stationary passage.

Since the blade velocity is always in the tangential direction, the axial velocities are unchanged.

$$
W_{z}=V_{z}
$$

In the coordinate system used the tangential velocity at the rotor entrance $(-100$ percent Chord) is zero. At the rotor exit $(-157.23$ percent Chord) the tangential velocity is $-155 \mathrm{~m} / \mathrm{s}$. The relative tangential velocity is

$$
W_{\theta}=V_{\theta}+r|\omega|
$$

Relative Mach Number

Using basic thermodynamic equations for the specific heat, the speed of sound, the work done by the system, and the energy equation, a quantity called the local speed of sound, $a_{\ell}$, is defined as follows:

$$
a_{1}=\left[a_{0}^{2}+(\gamma-1)\left(-\Delta V_{\theta} \Gamma \omega-\frac{V_{a b s}^{2}}{2}\right)\right]^{1 / \overline{2}}
$$

where

$$
V_{a b s}=\left(V_{z}^{2}+V_{\theta}^{2}\right)^{1 / 2}
$$

and $a_{0}$ is the speed of sound based on the plenum total temperature, $r$ is the radius, $\gamma$ is the ratio of the specific heat at constant pressure to the specific heat at constant volume, $w$ is the rotational speed, and $\Delta v_{\theta}$ is the change in tangential velocity from the plenum to the point of interest. This local speed is only an approximation since radial velocity components are neglected in calculating the absolute velocity. 
The magnitude of the relative velocity is

$$
W=\left(W_{z}^{2}+W_{0}^{2}\right)^{1 / 2}
$$

The relative Mach number is the given by

$$
M_{r e I}=\frac{W}{a_{l}}
$$

\section{Appendix B - Using PLOT3D}

\section{Measurement grid}

The measurement grid is 3 -dimensional and is represented in radial, $r$, circumferential, $\theta$, and axial, $z$, coordinates. However, the data was principally collected in the cascade plane which is a plane defined by $z$ and $\theta$ coordinates for nearly constant $r$. The measurement grid thus obtained is unrolled by the transformation

$$
\begin{aligned}
& x=z \\
& y=r(z) \theta
\end{aligned}
$$

\section{Flow Field}

To use PLOT3D to analyze the results from a computational fluid dynamic simulation one must have as input to the PLOT3D program the computation grid and values at each grid point of the flow field vector $\vec{Q}$ where the components of $Q$ are the density $\left(Q_{1}=\rho\right)$, the three compoents of momentum $\left(Q_{2}=\rho v_{x}, Q_{3}=\rho v_{y}, Q 4=\rho v_{z}\right)$, and the stagnation energy per unit volume $\left(Q_{5}=\rho \mathrm{c}_{\mathrm{v}} \mathrm{T}_{0}\right)$.

Since PLOT3D is to be used to visualize 2-dimensional information in a frame of reference rotating with the blade, the flow field vector, $\vec{Q}$, must be specified in terms of the relative velocities.

The mapping used to specify $\vec{Q}$ was chosen based on Eq. (17) which was used to define the grid. 
The following relations are used to specify these quantities in the rotating blade coordinate system:

$$
\begin{gathered}
Q_{1}=\rho_{\circ} \\
Q_{2}=\rho \mathrm{W}_{z} \\
Q_{3}=\rho \mathrm{W}_{\theta} \\
Q_{4}=0.0 \\
Q_{5}=\rho\left(\frac{C_{p} T_{l}}{\gamma}+\frac{W^{2}}{2}\right)
\end{gathered}
$$

where the relative velocity $w$, is given by Eq. (15), the relative tangential velocity, $W_{\theta}$, is given by Eq. (12), the local temperature, $T_{\ell}{ }^{\prime}$ is

$$
\mathrm{T}_{\ell}=\frac{{ }_{\omega{ }^{2}{ }_{\ell}}}{\gamma \mathrm{R}}
$$

the local acoustic velocity, $a_{\ell}$, is given by Eq. (13), $\gamma$ is the ratio of specific heats, $R$ is the universal gas constant, $8.314 \mathrm{~J} /(\mathrm{gm}$ mole $\mathrm{K}$ ), and $M_{\omega}$ is the molecular weight of air, 0.02897 $\mathrm{gm} /(\mathrm{kgm}$ mole $)$.

Using basic thermodynamic equations for the specific heat, the speed of sound, the work done by the system, and the energy equation, we find the local stagnation temperature to be given by

$$
c_{p}\left(T_{s}\right)_{l}=c_{p} T_{l}+\frac{1}{2} V_{a b s}^{2}=c_{p} T_{0}-\Delta V_{\theta} r \omega
$$

or

$$
\left(T_{a}\right)_{l}=T_{l}+\frac{1}{2}\left(\frac{\gamma-1}{\gamma}\right) \frac{M_{\omega}}{R} V_{a b s}^{2}=T_{0}-\left(\frac{\gamma-1}{\gamma}\right) \frac{M_{\omega}}{R} \Delta V_{\theta} r \omega
$$


The stagnation temperature, $\left(\mathrm{T}_{s}\right)_{\ell}{ }^{\prime}$ is also called the total temperature. It is the temperature attained when a gas at static local temperature, $\mathrm{T}_{\ell}$, and velocity, $\mathrm{V}_{\text {abs }}$, are brought to rest adiabatically.

The measured initial static pressure, $\left(p_{s}\right)_{\text {inlet' }}$ and the stagnation temperature, $\left(T_{s}\right)_{\text {inlet }}$. defined above are used as initial conditions. We assume that

$$
\begin{aligned}
& \left(p_{s}\right)_{\text {inlet }}=\left(p_{s}\right)_{\ell} \\
& \left(T_{s}\right)_{\text {inlet }}=\left(T_{s}\right)_{\ell}
\end{aligned}
$$

The measured initial pressure, $\left(p_{s}\right)_{\text {inlet' }}$ is regarded at the pressure of a fluid brought to rest isentropically from a velocity $V_{\text {abs }}$ and a static local pressure $p_{\ell}$. Thus

$$
\frac{p_{l}}{\left(p_{s}\right)_{\text {inlet }}}=\left[\frac{T_{l}}{\left(T_{s}\right)_{\text {inlet }}}\right]^{\frac{\gamma}{(\gamma-1)}}
$$

From static temperature, $T_{\ell}$, and static pressure, $p_{\ell}$, the density $\rho_{\ell}$ is calculated using the ideal gás equation.

Between points along a streamline the flow process is considered to be a polytropic process so that the pressure and temperature at these points are related as follows:

$$
\frac{p_{l}+1}{p_{l}}=\left(\frac{T_{l}+1}{T_{l}}\right)^{\frac{n}{n-1}}
$$

Again at each local point, using $T_{\ell}$ and $\mathrm{P}_{\ell}$, the density $P_{\ell}$ is calculated with the ideal gas equation. This local density is used to determine the input for PLOT3D.

In addition to the calculations of the PLOT3D input, the total or stagnation pressure is 
calculated at each local point assuming an isentropic process:

$$
\frac{\left(p_{s}\right)_{l}}{\left(p_{l}\right)}=\left(\frac{\left(T_{s}\right)_{l}}{T_{l}}\right)^{\frac{\gamma}{(\gamma-1)}}
$$

The pressure, temperature, and density calculated from the laser measurements are an approximation to the true pressure, temperature, and density since only two of the three components of velocity are available. Thus, the Mach number is also an approximation to the true Mach number.

\section{Appendix C - Software}

Computer programs were developed or were available to perform each of the four functions. The prime impediment in implementing the MMS procedure was the need to combine these programs in a single system. For the benefit of anyone who must implement a MMS procedure the system logic which was developed will be discussed. In this discussion the names of the data files and Fortran programs will be used. Figure $\mathrm{C}-1$ shows a logic diagram of the files and three FORTRAN software programs used to put the procedure into practice. Note that two of the three programs are related to grid generation. The input data files contain the axial-flow fan rotor geometry information (SURF10.DAT) and the 2-dimensional laser anemometry information (ST_NS30.DAT and SD_NS30.DAT).

Three software programs are used. The first program (INFLAPO1.FOR) computes, using spline curve fits if necessary, the input boundary information for the grid generation program (FOIL2.FOR). It produces files with status information that are used to study the behavior of the program (INFLAPO1.OUT), the grid information needed by the third program (GRID1.DAT), and input information for the second program which generates the monitor surface grid (AIRFOIL5.DAT). 
The second program (FOIL2.FOR) generates the monitor surface grid (GRID1.X,Grid1.Q) using a successive over-relaxation (SOR) to solve the partial differential equation that defines the grid. In addition, it produces files with status information that indicate the rate of convergence of the SOR iteration procedure (AIRFOIL5.OUT) and the initial grid guess (INOUT1.X, INOUT1.Q). The monitor surface data sets GRID1.0 $Q$ and INOUT1.Q are empty. However, the (INOUT1.X, INOUT1.Q) and (GRID1.X, GRID1.Q) file pairs can be viewed using PLOT3D to see if the new grid is satisfactory and assess the starting grid.

The third program (COMBINE2.FOR) combines the measured grid information, the measured 2-dimensional velocities, the physical model, the experiment test conditions, and the new grid, to produce the monitor surfaces of density 2-dimensional momentum, and energy (BINDEA.X, BINDEA.Q) using Shepard's method to interpolate between the monitor surface grid and the measurement grid. This program also produces monitor surfaces on the original measured grid (BINDED. $X$, BINDED.Q) and status information (COMBINE.OUT).

Each of these programs has a specific data file with control information (INFLAP01.DAT, FOIL2.DAT, and COMBINE2.DAT). In addition, there is a master data file with control information (MASTER.DAT). These control files perform functions such as specifying the number of points in the monitor surface grid, the grid cluster control parameters, and the weighting procedure to be used by Shepard's method. 


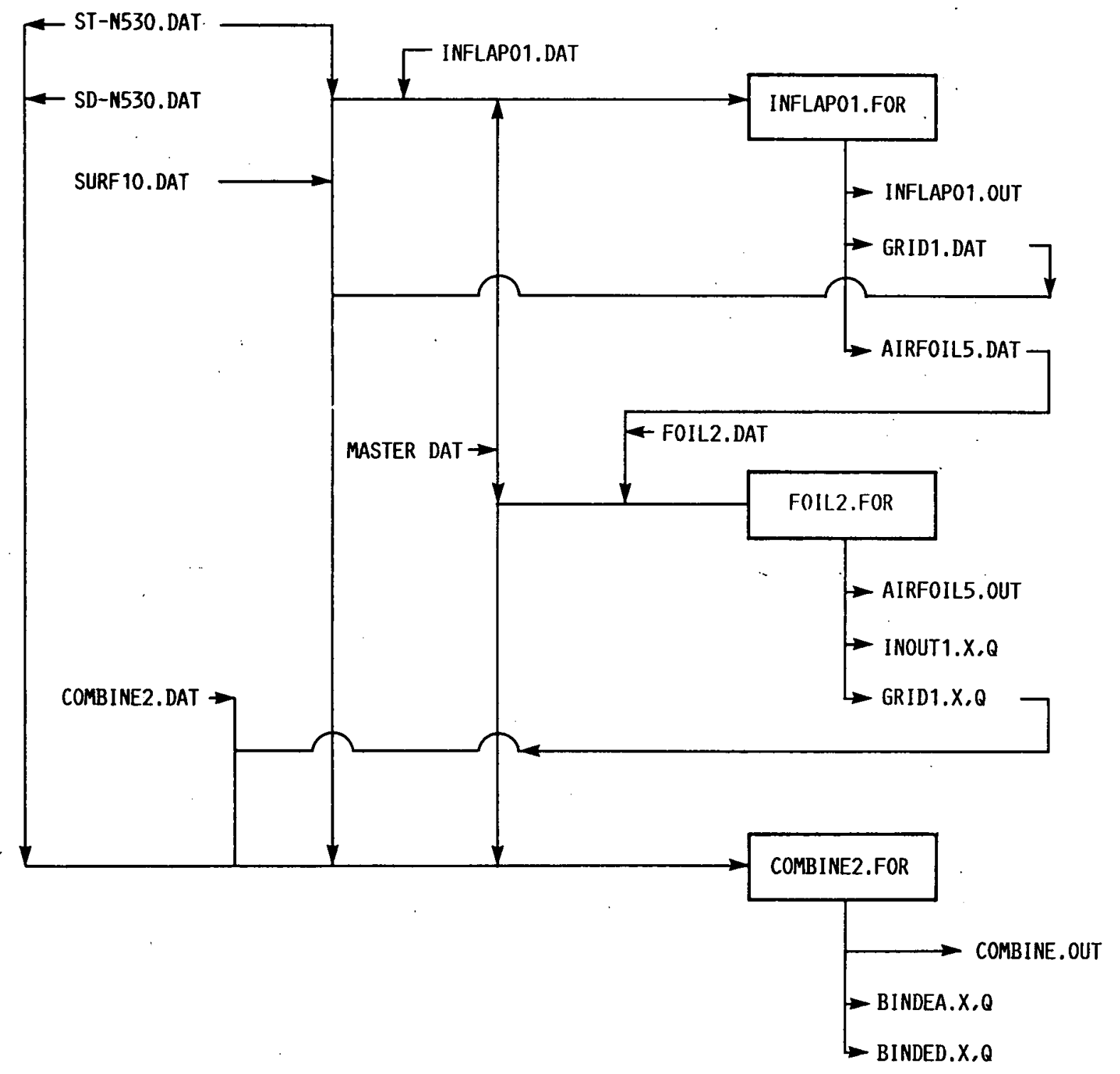

FIG. C-1. - BLOCK DIAGRAM OF COMPUTER PROGRAM LOGIC. 


\section{$\underline{\text { References }}$}

1. Buning, P. and Steger, J., "Graphics and Flow Visualization in Computational Fluid Dynamics," AIAA Paper 85-1507-CP; July, 1985.

2. Lasinski, T., Buning, P., Choi, D., Rogers, S., Bancroft, G., and Merritt, F., "Flow Visualization of CFD Using Graphics. Workstations," AIAA Paper 87-1180, June, 1987.

3. Bancroft, G., Merritt, F., Buning, P., and Watson, V., "High Definition Graphics Application in Fluid Flow Simulations," in True 3D Imaging Techniques and Display Technologies; Proceeding of the Meeting, Los Angeles, CA., Jan. 15-16, 1987, edited by D.F. MCAllister and W.Z. Robbins, SPIE, 1987, pp. 157-162. (SPIE Proceedings, Vol. 761, 1987).

4. Strazisar, A.J., Wood, J.R., Hathaway, M.D., and Suder, K.L., "Laser Anemometer Measurements in a Transonic-Axial-Flow Fan Rotor," NASA TP-2879, 1989.

5. Wood, J., Strazisar, A.J., and Simonyi, P.S., "Shock structure Measured in a Transonic Fan. Using Laser Anemometry," in AGARD Transonic and Supersonic Phenomena in Turbomachines, AGARD CP-401, 1987.

6. Walatka, P.P., and Buning, P.G., "PLOT3D User's Manual," NASA TM-101067, 1989.

7. Thompson, J.F., Thames, F.C., and Mastin, C.W., - Tomcat - a Code for Numerical Generation of Boundary-Fitted Curvilinear Coordinate systems on Fields Containing any Number of Arbirary Two-Dimensional Bodies," Journal of Computational Physics, Vol. 24, July 1977, pp. 274-302.

8. Thompson, J.F., Thames, F.C., and Mastin, C.W., "Boundary-Fitted Curvilinear Coordinate Systems for solution of Partial 
Differential Equations on Fields Containing any Number of Arbitrary Two-Dimensional Bodies," NASA CR-2729, 1977.

9. Fletcher, C.A.J., Computational Techniques for Fluid Dynamics, Vol. 2, Springer-Verlag, 1988.

10. Thompson, J.F., Warsi, Z.U.A., and Mastin, C.W., Numerical Grid Generation, North Holland/Elsevier Science Publishing Co., 1985.

11. Lancaster, P., and Salkauskas, K., Curve and Surface Fitting, Academic Press, 1986.

12. Alfeld, P., Scattered Data Interpolation in Three or More Variables, "in Mathematical Methods in Computer Aided Geometric Design, edited by T. Lyche and L.L. Schumaker, Academic Press, 1989, pp. 1-33.

13. Schumaker, L.L., "Fitting surfaces to scattered Data," in Proceedings of the NASA Workshop on Surface Fitting, edited by L.F. Guseman, Jr., NASA, 1982, pp. 27-94 (also NASA CR-167779, 1983).

14. Franke, R., "Scattered Data Interpolation: Tests of Some Methods," Mathematics of Computation, vol. 38, No. 157, 1982, pp. 181-200.

15. Van Wylen, G.J., and Sonntag, R.E., Fundamentals of classical Thermodynamics, 3rd ed., SI Version, John Wiley \& Sons, 1985 .

16. Glassman, A.J., ed., Turbine Design and Application, Vol. 1, NASA SP-290, 1972.

17. Jennings, B.H., and Rogers, W.L., editors. Gas Turbine Analysis and Practice, Dover Publications, 1969. 


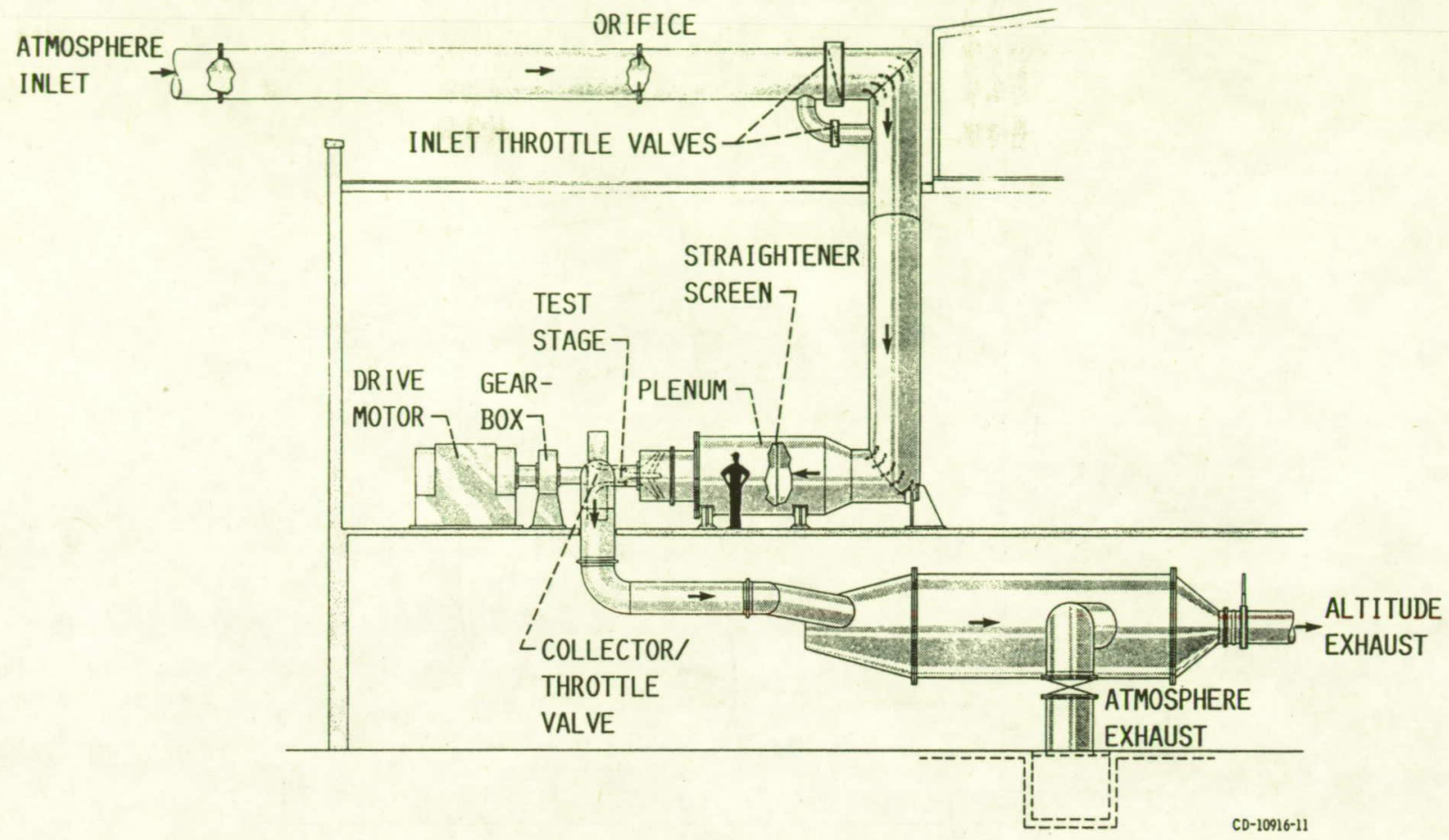

FIG. 1. - NASA LEWIS SINGLE-STAGE COMPRESSOR TEST FACILITY.

\section{ORIGINAL PAGE \\ BLACK AND WHITE PHOTOGRAPH}

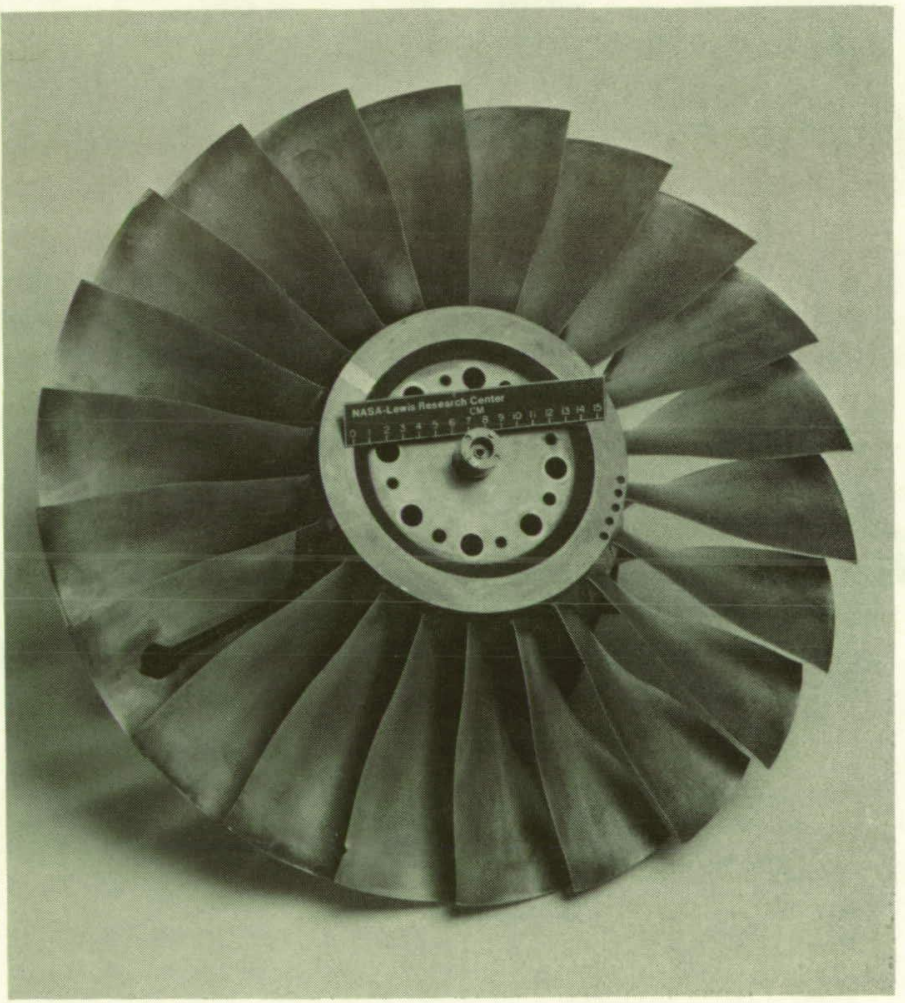

FIG. 2. - TEST ROTOR.
LA OPIICAL AXISᄀ

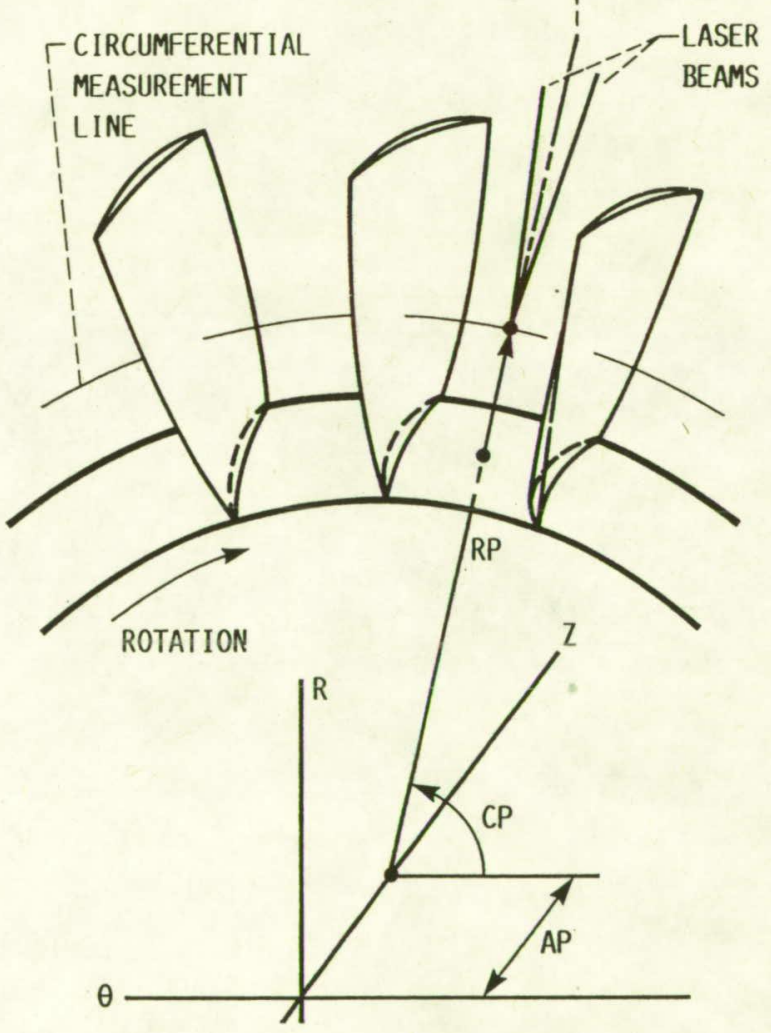

FIG. 3. - DEFINITION OF LASER ANEMOMETER CIRCUMFERENTIAL MEASUREMENT LINE. 


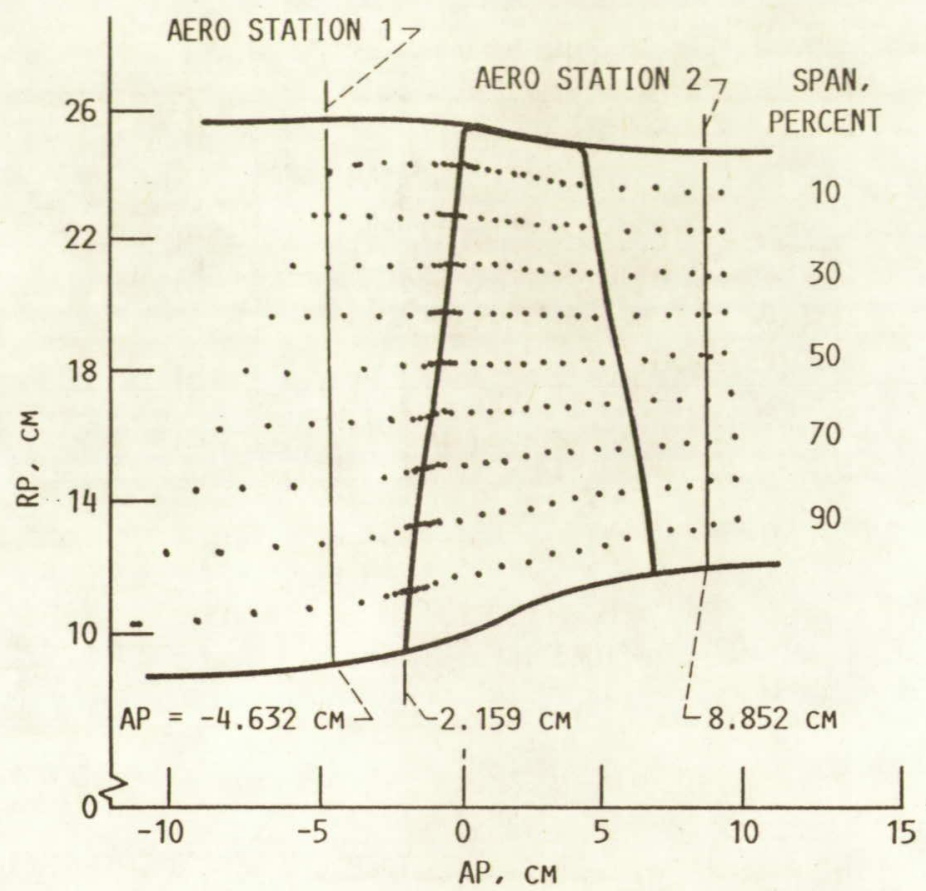

FIG. 4. - MERIDIONAL VIEW OF TEST FAN ROTOR SHOWING LASER ANEMOMETER AND AERODYNAMIC SURVEY LOCATIONS.

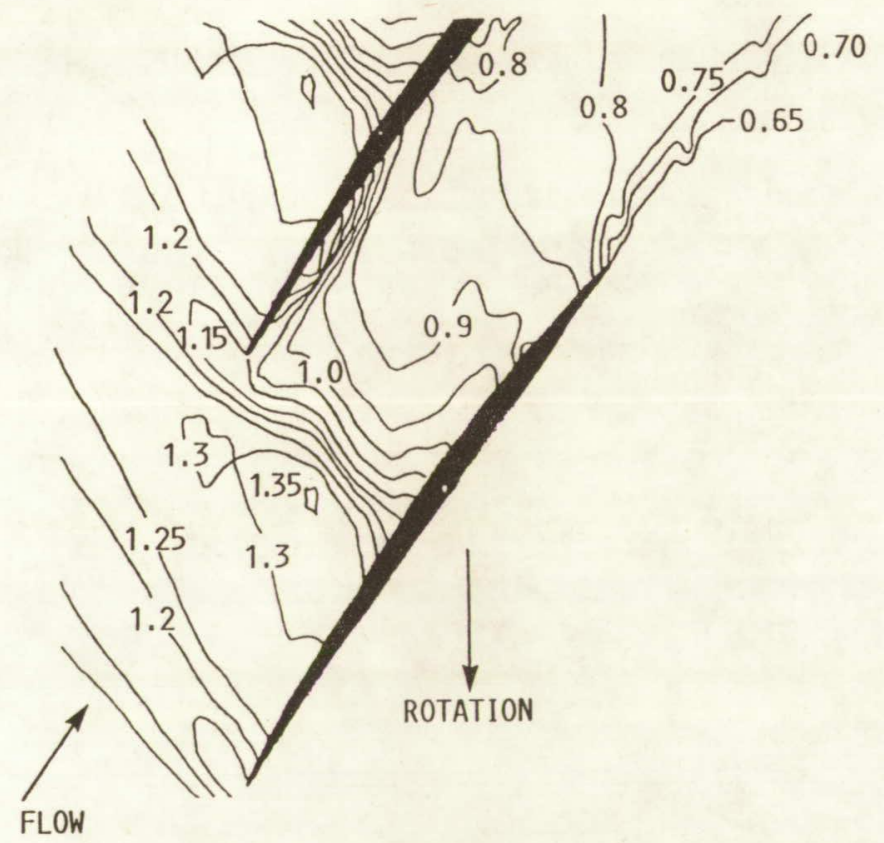

FIG. 5. - CONTOUR PLOT OF RELATIVE MACH NUMBER AT NEARSTALL FLOW TEST CONDITION AT 30-PERCENT OF THE SPAN FROM THE SHROUD. 


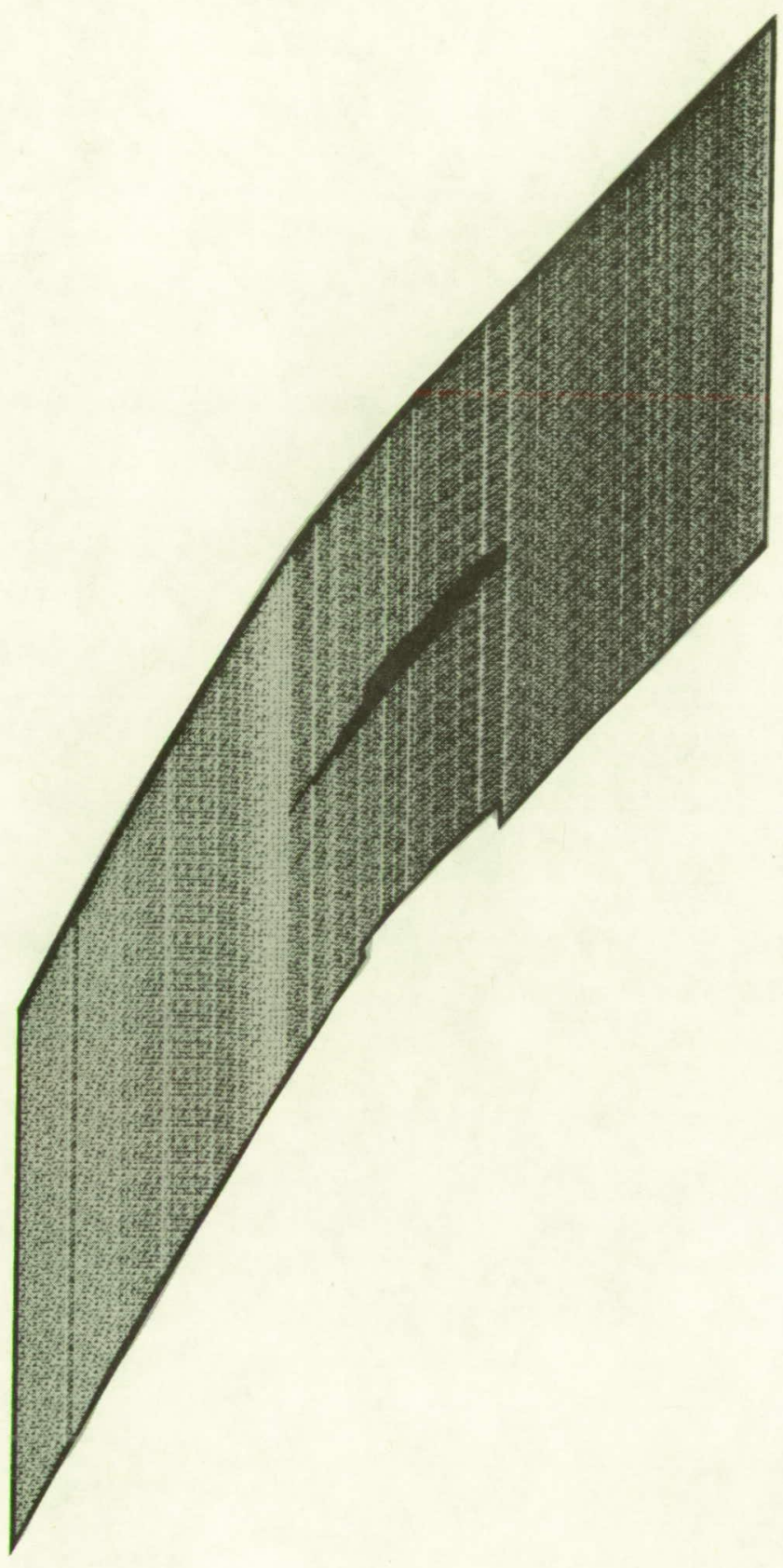

FIG. 6. - MEASUREMENT GRID.

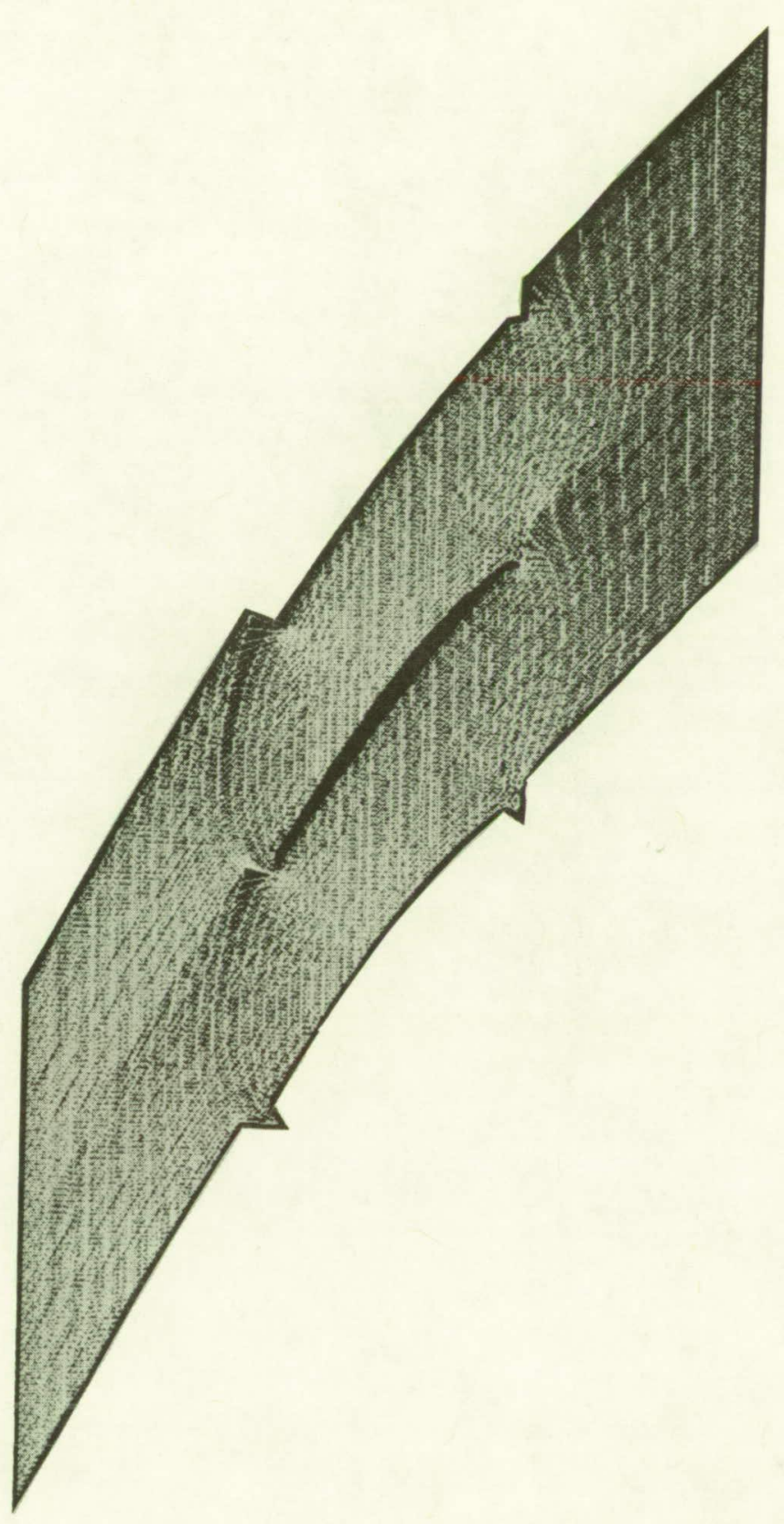

FIG. 7. - MONITOR SURFACE GRID. 


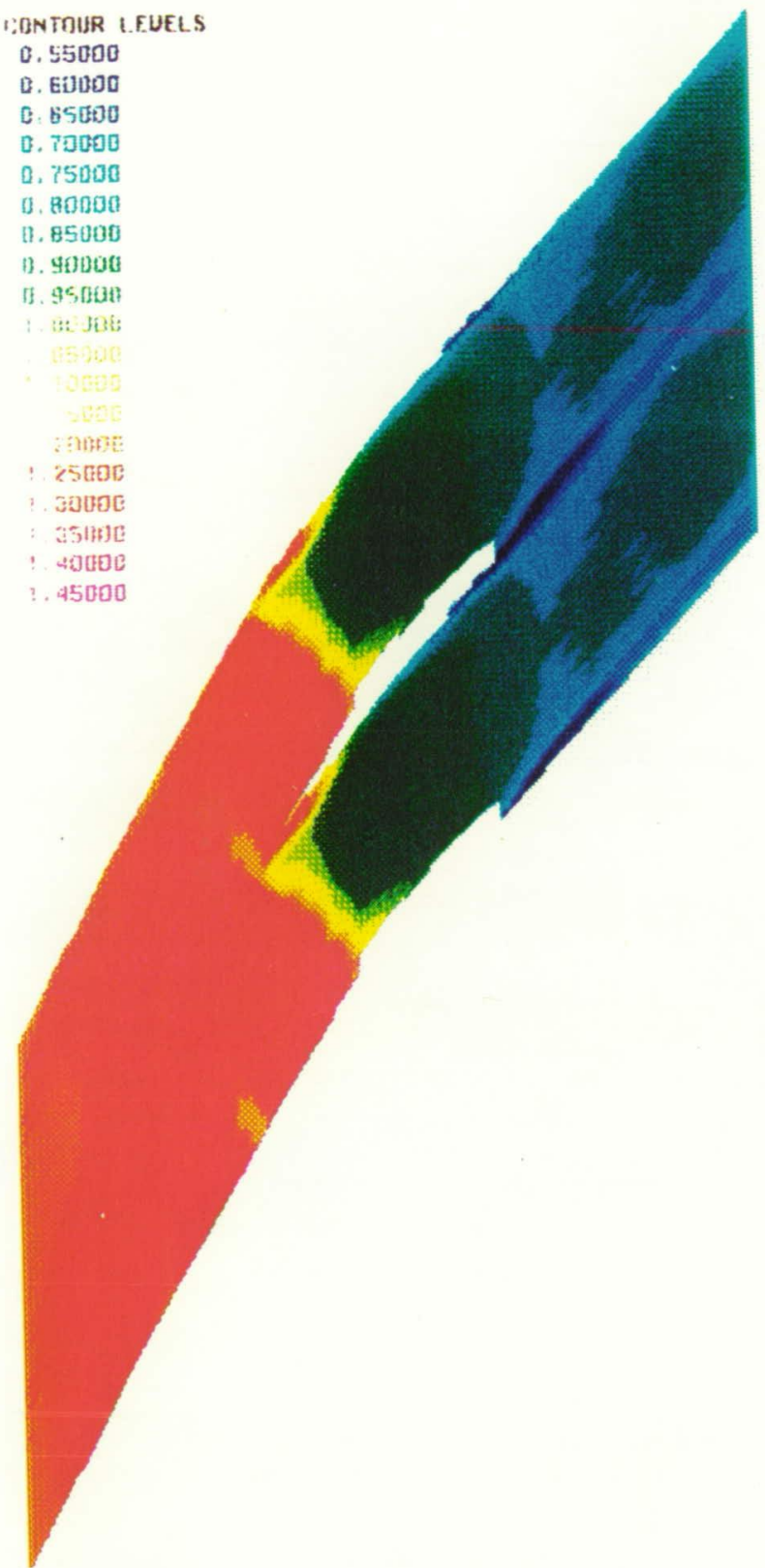

FIG. 8. - SHADED SURFACE RELATIVE MACH NUMBER PLOT BASED ON MEASURED DATA.

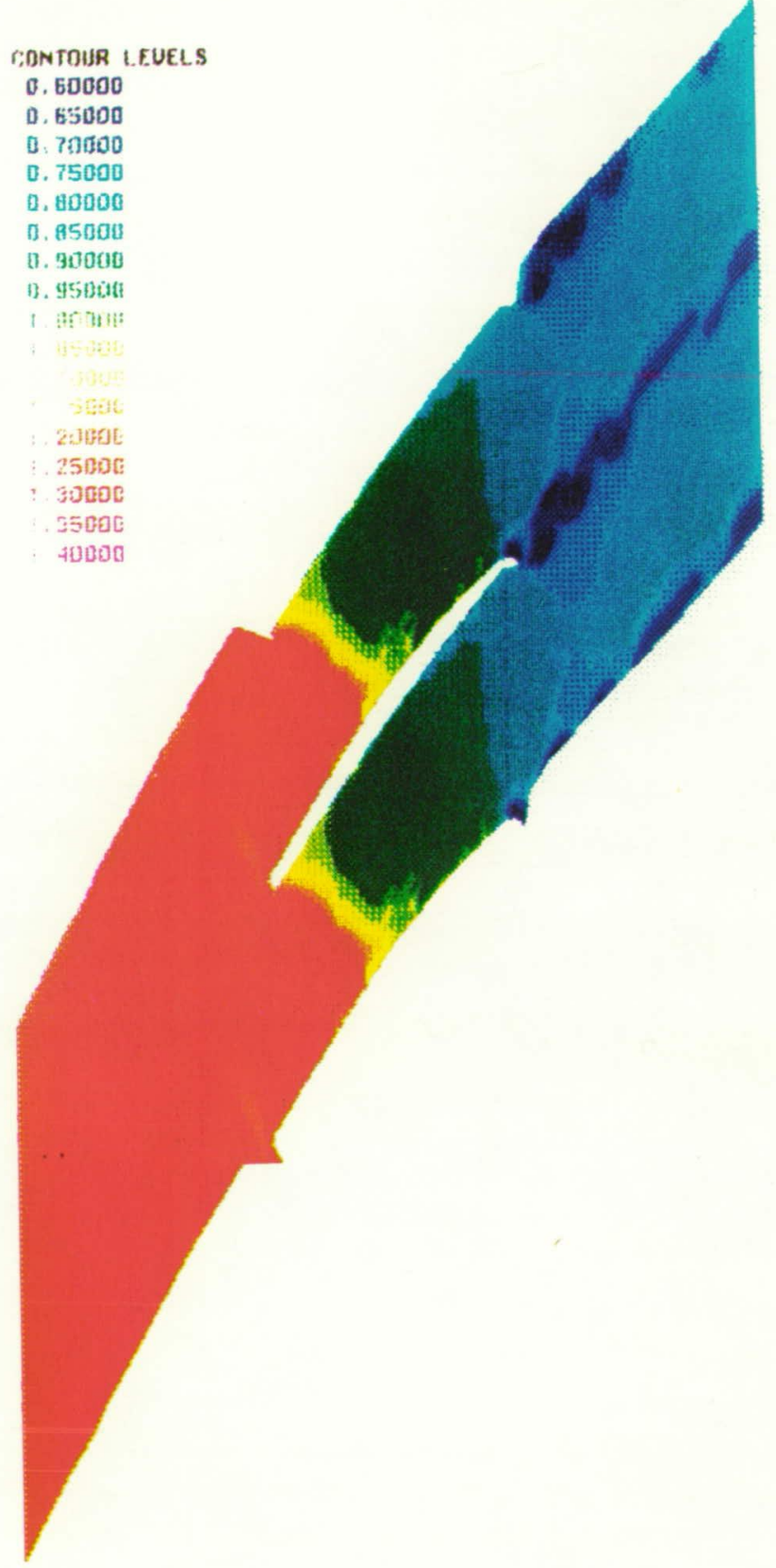

FIG. 9. - SHADED SURFACE RELATIVE MACH NUMBER PLOT BASED ON MONITOR SURFACES. 
0. 70000

a. 75000

1]. $80000 n$

6. 850015

0. 9obau

15. $9506 \mathrm{at}$

. :3:

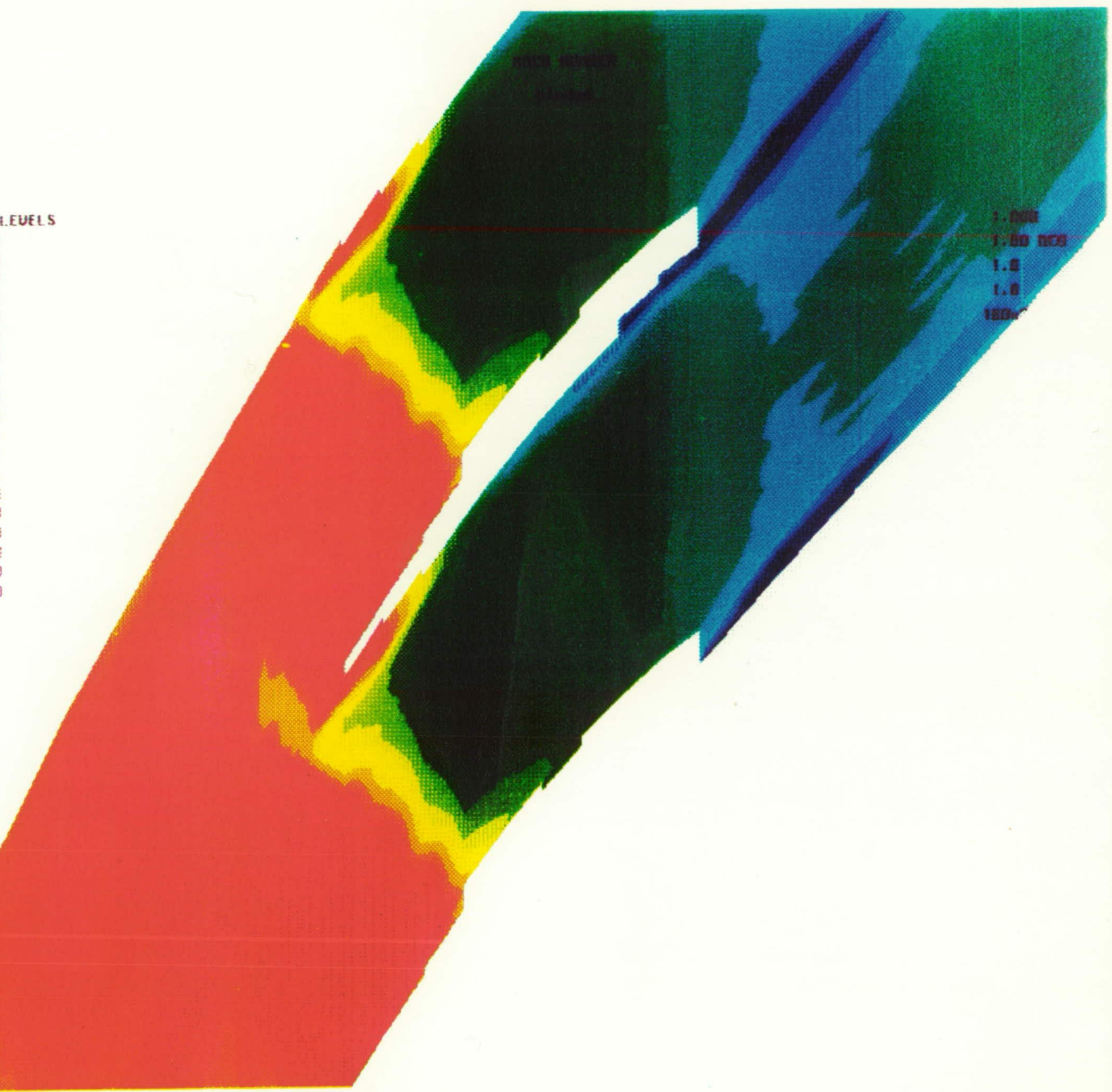

Fig. 10. - ClOSEUP VIEW OF SHADEd SURFACE RELATIVE MACH NUMBER PLOT BASED ON MEASURED DATA. 


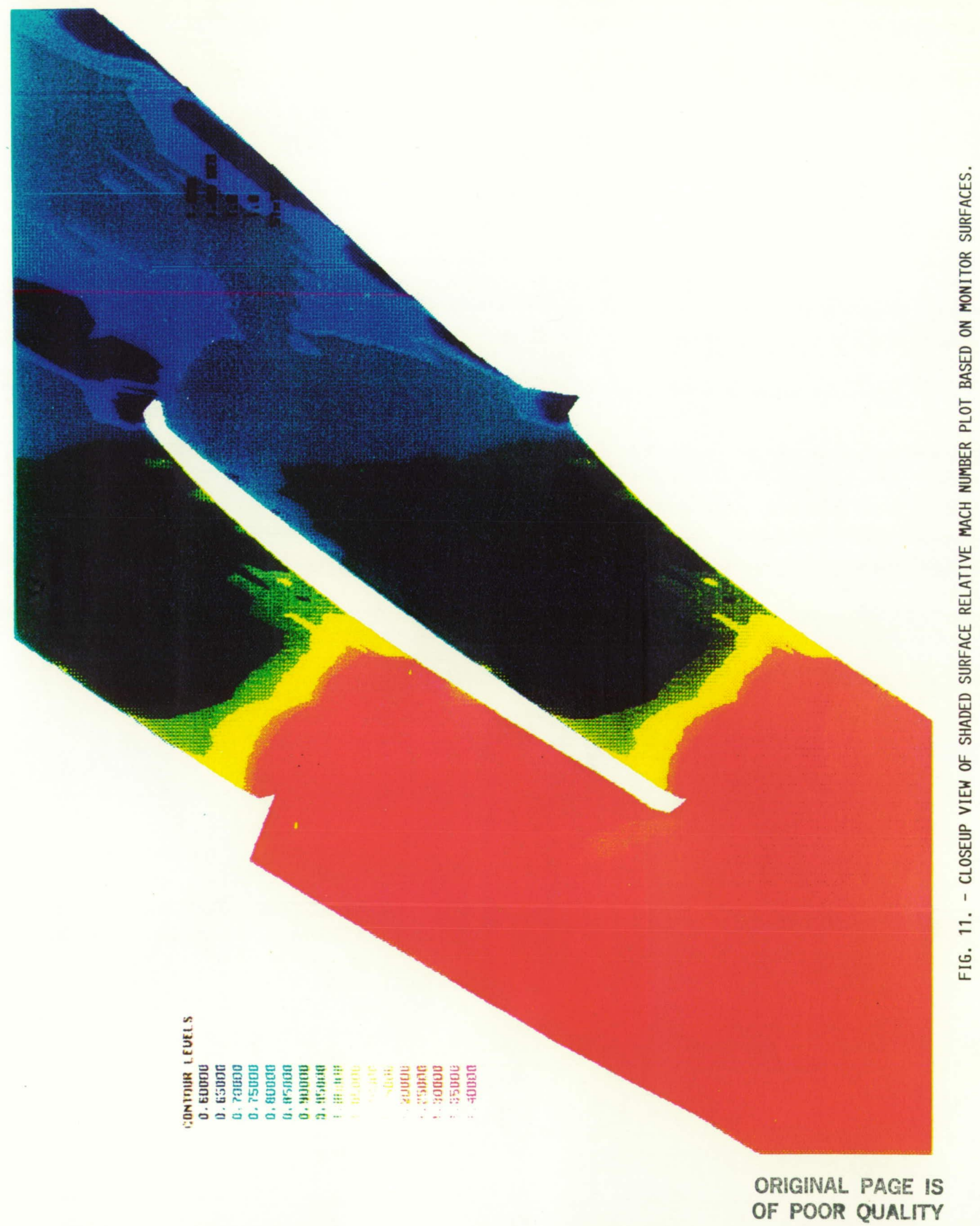




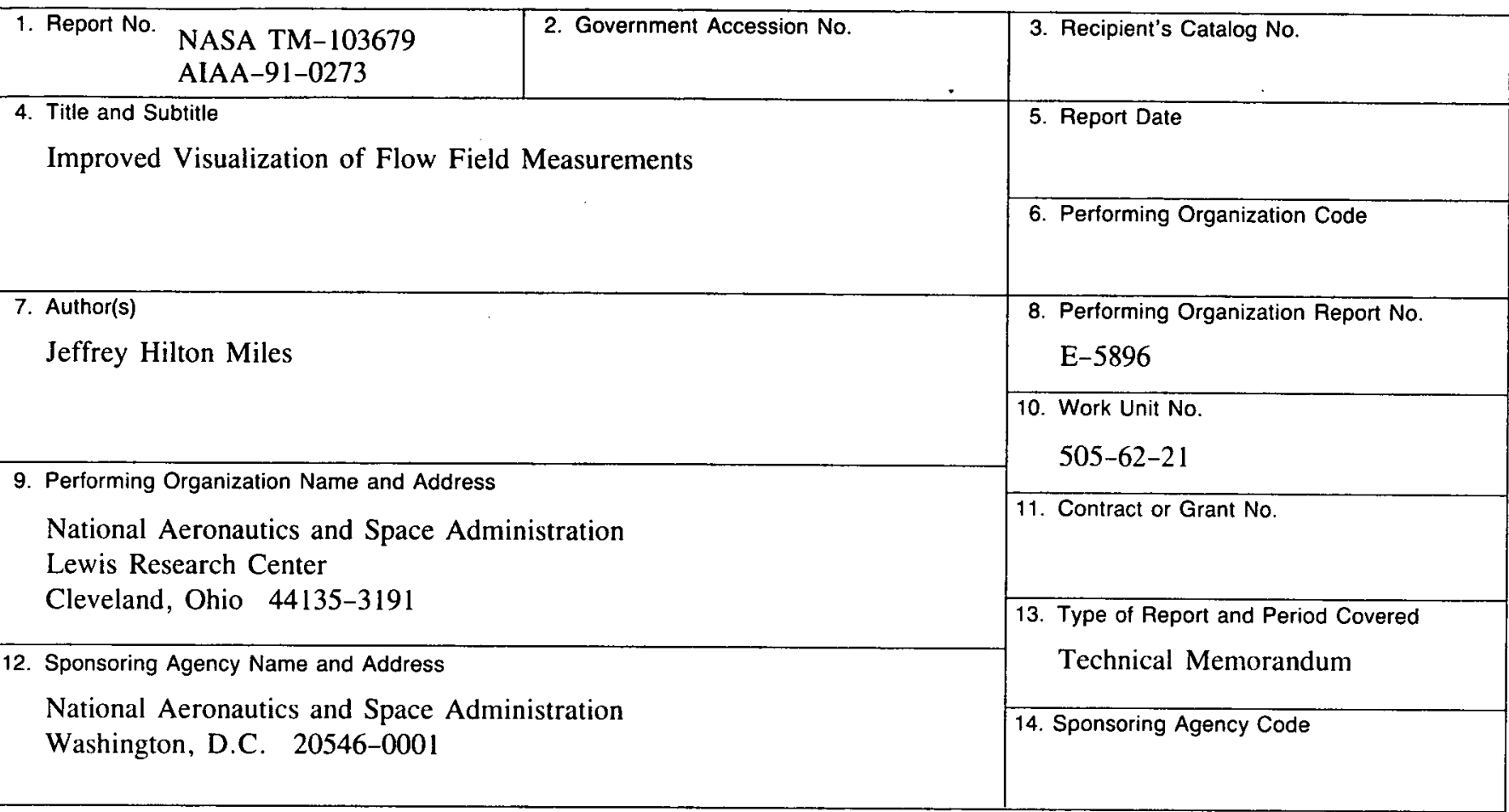

15. Suppiementary Notes

Prepared for the 29th Aerospace Sciences Meeting sponsored by the American Institute of Aeronautics and Astronautics, Reno, Nevada, January 7-10, 1991.

16. Abstract

A capability thias been developed that makes it possible to apply to measured flow field data the visualization tools developed to display numerical solutions for computational fluid dynamic problems. The measurement monitor surface (MMS) procedure was applied to the analysis of flow field measurements within a low-aspect-ratio transonic axial-flow fan rotor obtained with 2-dimensional laser anemometry. The procedure generates input for the visualization tools developed to display numerical solutions for computational fluid dynamic problems. The relative Mach number contour plots obtained by this method resemble the conventional contour plots obtained by more traditional methods. The results demonstrate that the MMS procedure can be used to generate input for the multidimensional processing and analysis tools developed for data from numerical flow field simulations. They show that an experimenter can apply the MMS procedure to his data and then use an interactive graphics program to display scalar quantities like the Mach number by profiles, carpet plots, contour lines, and surfaces using various colors. In addition, flow directionality can be illustrated by display of vector fields and particle traces. The MMS procedure thus has the potential to produce better insight into turbomachinery and other types of fluid flow physics.

17. Key Words (Suggested by Author(s))

Turbomachinery

Flow visualization

Monitor surface

Laser anemometry
18. Distribution Statement

Unclassified - Unlimited

Subject Category 02

19. Security Classif. (of this report)

$$
\text { Unclassified }
$$

20. Security Classif. (of this page)
Unclassified
21. No. of pages

37
22. Price*

A03 